\title{
Approximations for Aligned Coloring and Spillage Minimization in Interval and Chordal Graphs
}

\author{
Douglas Carroll $^{1}$, Adam Meyerson ${ }^{2}$, and Brian Tagiku ${ }^{2}$ \\ 1 Raytheon Company. E-mail: decarroll@raytheon.com \\ 2 University of California, Los Angeles. E-mail: \{awm, btagiku\}@cs.ucla.edu
}

\begin{abstract}
We consider the problem of aligned coloring of interval and chordal graphs. These problems have substantial applications to register allocation in compilers and have recently been proven NP-Hard. We provide the first constant approximations: a $\frac{4}{3}$-approximation for interval graphs and a $\frac{3}{2}$-approximation for chordal graphs. We extend our techniques to the problem of minimizing spillage in these graph types.
\end{abstract}

\section{Introduction}

One of the most complex and time-consuming aspects of a compiler is the register allocation process where variables are assigned to registers. The implementation of the register allocator is of upmost importance as it has a substantial impact on the efficiency of code generated [10]. The seminal works of Chaitin et al. [8, 7] established a connection between register allocation and graph coloring. Since these results, a number of heuristic techniques based on splitting of live ranges and hierarchical divide and conquer have been proposed $[9,13,6,5,4]$. However, provably good approaches have eluded researchers, primarily because graph coloring in the general case is NP-Hard and also difficult to approximate [15].

Despite these hardness results, graph coloring is more tractable on special classes of graphs. Two examples are the class of chordal graphs and its subclass of interval graphs, for which coloring can be computed optimally in linear time [12]. These graphs arise in many applications and, fortunately, many real programs do in fact correspond to chordal and interval graphs $[17,14]$. Thus, algorithmic results for these classes are meaningful to register allocation and a broad range of other applications.

Modern work in register allocation $[3,2,1,20,18,16]$ now focuses on heterogeneous register architectures where variables are either single-word (requiring one register) or double-word (requiring two registers). Double-word variables must be stored in adjacent registers starting on an even address (i.e. the registers must be aligned). Indeed, most modern architectures are heterogeneous which stresses the importance of this model. The corresponding coloring problem is called the aligned coloring problem, introduced by Lee, Palsberg, and Pereira [16] who prove it to be NP-Hard even when restricted to chordal or interval graphs. We 
give the first approximation results for aligned coloring: a $\frac{4}{3}$-approximation for interval graphs and a $\frac{3}{2}$-approximation for chordal graphs.

We also consider the problem of minimizing spillage both in the aligned and unaligned case. Here, we are given a specific number of colors (or registers) and asked to color as many graph nodes as possible subject to this constraint. This problem relates directly to register allocation since the number of colors (registers) is a fixed property of the machine architecture. The unaligned problem is known to be NP-Hard even for chordal graphs [21]. We show that it is hard to approximate to $\Omega(\log n)$ based on a reduction from set cover [11] and give a matching $O(\log n)$-approximation. If we are permitted to run in time exponential in the number of colors, there is an exact algorithm for chordal graphs with running time $O\left(n m(\tau+1)^{c}\right)$ for $c$ colors, $n$ nodes, $m$ edges, and tree-width $\tau$. We show that if we are willing to accept a $1+\epsilon$ approximation, we can improve this running time to $O\left(n m\left(c+\frac{c}{\epsilon}\right)^{c}\right)$ (independent of the tree-width).

For the aligned version over chordal graphs, we can apply dynamic programming to get a $1+\epsilon$ approximation in running time $O\left(n m(c+1)^{c+c / \epsilon}\right)$. If we are willing to accept a bicriteria approximation in which we use extra colors to obtain at most the spillage needed by optimum using $c$ colors, then we can give bounds of $\left(\frac{3}{2}, \log n\right)$ and $\left(\frac{4}{3}, 1\right)$ for chordal and interval graphs respectively, where the first factor is on the number of colors and the second is on the spillage.

\section{Preliminaries and Notation}

Our work will be restricted to the class of chordal and interval graphs. A graph is chordal if every cycle of four or more vertices has a chord (an edge joining two non-adjacent vertices in the cycles). A graph is interval if its vertices correspond to intervals of the real line and two vertices are adjacent if and only if their corresponding intervals intersect. For convenience, we will sometimes express an interval graph using a collection of intervals rather than show the graph representation.

We consider a generalization of graph coloring in which certain vertices require two colors instead of just one. Thus, graphs $G=(V, E)$ will have vertex weights $w: V \rightarrow\{1,2\}$ indicating the number of colors a vertex requires. We will call these 1,2-vertex-weighted graphs. We extend the notion of clique number $\omega(G)$ to be the maximum total weight of any clique of $G$. We represent colors numerically starting with color 0 and any vertex requiring two colors also requires that the colors are $2 i$ and $2 i+1$ for integer $i$. Formally:

Definition 1 (Aligned $c$-Coloring). An aligned $c$-coloring for a 1,2-vertexweighted graph $G=(V, E)$ with weights $w$ is a mapping $\phi: V \rightarrow\{0, \ldots, c-1\}$ such that for all $(u, v) \in E$ we have $\phi(u) \neq \phi(v)$. Additionally, if $w(u)=2$, then $\phi(u)$ must be even and for all $(u, v) \in E$ we must have $\phi(u)+1 \neq \phi(v)$. This corresponds to assigning any vertex $u$ with $w(u)=2$ to two consecutive colors starting from an even value.

At times we will say weight- 1 vertex $v$ blocks color $2 i$ (and accordingly, $2 i$ is blocked by $v$ ) if $\phi(v)=2 i$ or $\phi(v)=2 i+1$. Figure 1 gives examples of aligned 
and unaligned colorings of a collection of intervals of time (thus, corresponding to an interval graph). Note that Figure 1 also shows that an aligned coloring may require more than $\omega(G)$ colors.

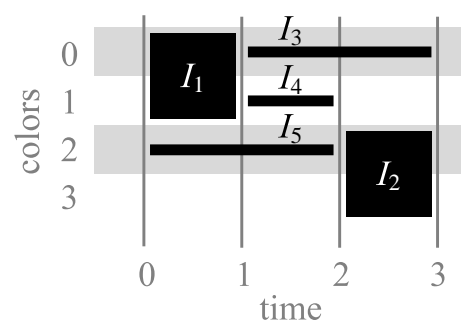

(a)

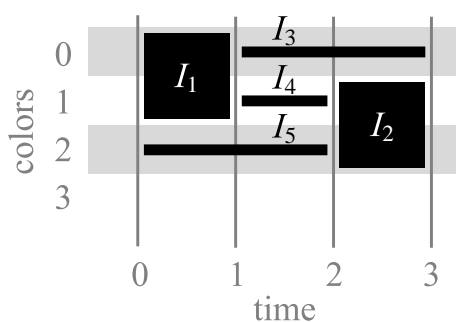

(b)

Fig. 1. Colorings of a collection of intervals of time. Thick intervals have weight 2 while thin intervals have weight 1. (a) A proper aligned coloring. (b) A minimum unaligned coloring.

We use $a \chi(G)$ to denote the aligned chromatic index of $G$ (i.e., the smallest $c$ for which $G$ is aligned $c$-colorable). Note that we can easily lower bound $a \chi(G)$ using the clique number of $G$ :

$$
\omega(G) \leq a \chi(G) .
$$

For any integer $k \geq 0$, we let $a \chi(k)$ denote the maximum aligned chromatic index over all 1,2-vertex-weighted graphs $G$ with $\omega(G)=k$.

\section{The Aligned Coloring Problem}

Problem 1 (Aligned Coloring Problem). Given 1,2-vertex-weighted graph $G=(V, E)$, find an aligned c-coloring $\phi$ of $G$ where $c$ is minimal.

In this section we give approximations for the aligned coloring problem. Since the problem is hard to approximate for general graphs [15], we restrict our attention to either chordal graphs or interval graphs. Although standard minimum coloring can be solved in linear time for either class [12], the aligned coloring problem remains NP-Complete [16]. It is straightforward to produce a 2approximation for the problem by simply splitting the graph into two subgraphs based on vertex weights and then coloring each using a distinct set colors. We will provide the first approximation algorithms which improve upon this factor, giving a $\frac{3}{2}$-approximation for chordal graphs and a $\frac{4}{3}$-approximation for interval graphs.

\subsection{Chordal Graphs}

For any chordal graph $G$, we can produce a tree decomposition of minimum tree width in polynomial time [12]. Each set $X_{i}$ in this decomposition represents a 
clique, thus $w\left(X_{i}\right)=\sum_{v \in X_{i}} w(v) \leq \omega(G)$. We will color $G$ greedily by considering sets of the tree decomposition from the root downwards. For each set $X$, we consider all uncolored nodes in arbitrary order and assign the minimum color which does not conflict with any color of another member of $X$. We can show that this uses at most $\frac{3}{2} \omega(G)$ colors.

Theorem 1. The above algorithm successfully produces an aligned coloring using at most $\frac{3}{2} \omega(G)$ colors. Thus it is a $\frac{3}{2}$-approximation to the problem of aligned coloring of a chordal graph.

Proof. First we show that a valid aligned coloring is produced. Consider any edge $(u, v) \in E$. Let $X$ be the first tree decomposition set we consider which contains both $u, v \in X$. Since we consider sets from the root downwards and since we know that $u, v$ were not both in the parent of $X$, one of $u, v$ must be appearing for the first time. Thus, we can assign a color such that there are no conflicts.

Now we bound the number of colors. Consider any node $u$ with $w(u)=1$. Let $X$ be the tree decomposition set in which we first encounter $u$. Since $w(X) \leq$ $\omega(G)$, at least one of colors 0 through $\omega(G)-1$ must be available. Thus, all weight-1 vertices are assigned a color at most $\omega(G)-1$.

Now consider a node $u$ with $w(u)=2$ and let $X$ be the tree decomposition set in which we first encounter $u$. Suppose that there are $x_{1}$ weight- 1 nodes and $x_{2}$ weight-2 nodes (excluding $u$ ) in $X$. Then $w(X-u)=x_{1}+2 x_{2} \leq \omega(G)-2$. When we assign a color to $u$, suppose the color assigned were at least $\frac{3}{2} \omega(G)$. It would follow that since all nodes with $w(v)=1$ are assigned colors at most $\omega(G)$, we must have at least $x_{2} \geq \frac{1}{4} \omega(G)$ as otherwise we could have selected a smaller color. On the other hand, each node in $X$ can block at most one even color, so we must have $x_{1}+x_{2} \geq \frac{3}{4} \omega(G)$. Combining these two inequalities gives a contradiction.

\subsection{Chordal Graph Lower Bound}

There do in fact exist aligned chordal graph coloring problems where we need $\frac{3}{2} \omega(G)$ colors. This does not necessarily imply hardness of approximation, but it does indicate that we will need to use a different lower bound on optimum if we are to improve upon our $\frac{3}{2}$-approximation.

Fix $n>0$. We build a 1,2-vertex weighted graph $G_{n}$ with $\omega\left(G_{n}\right)=4 n$ as follows: $G_{n}$ will have a clique of $4 n$ weight- 1 vertices. Call this clique $K_{1}$ and let $V_{1}$ be its vertices. For each $S \subseteq V_{1}$ with $|S|=2 n$, we add a distinct clique $K_{S}$ of $n$ weight-2 vertices and all possible edges between vertices in $S$ and $K_{S}$. It is easy to check that $G_{n}$ is chordal and that $\omega\left(G_{n}\right)=4 n$.

Theorem 2. For each $n>0$, there exists no aligned coloring of $G_{n}$ using less than $\frac{3}{2} \omega\left(G_{n}\right)=6$ n colors.

Proof. Assume, by way of contradiction, that we have an aligned coloring of $G_{n}$ using at most $6 n-1$ colors. Consider $V_{1}$. These vertices form a clique so they 
must all be given unique colors. Then at least $2 n$ even colors are blocked by vertices in $V_{1}$. Let $X \subset V_{1}$ be a set of $2 n$ vertices that each block a distinct even color. Then notice that there are $n-1$ even colors that are not blocked by $X$. But then $K_{X}$ does not have enough even colors for its $n$ weight- 2 vertices. This contradicts the existence of our coloring and proves the claim.

\subsection{Interval Graphs}

We will show that every 1,2-vertex-weighted interval graph $G$ has an aligned coloring using $\frac{4}{3} \omega(G)$ colors. Our algorithm works by first decomposing $G$ into multiple smaller graphs, then approximately coloring each of the smaller graphs with disjoint sets of colors. We perform this decomposition as described below:

Theorem 3. For every integer $\alpha \geq 1$, every 1,2-vertex-weighted interval graph $G$ with $\omega(G)>\alpha+1$ can be decomposed (in polynomial-time) into 1,2-vertexweighted interval graphs $H$ and $G^{\prime}$ such that $\omega(H) \leq \alpha+1$ and $\omega\left(G^{\prime}\right)=\omega(G)-\alpha$.

Proof. Order the vertices of $G$ so that for each vertex $v$ all its neighbors appearing before it in the ordering form a clique (i.e. in a reverse perfect vertex elimination scheme). Such an ordering can be computed in linear time [19]. We partition the vertices of $G$ into $V_{H}$ and $V^{\prime}$ and let $H=G\left[V_{H}\right]$ and $G^{\prime}=G\left[V^{\prime}\right]$ (where $G[X]$ is the subgraph of $G$ induced by vertex set $X$ ). This is done by taking vertices one at a time in order. We add the next vertex to $V_{H}$ if it can be added without increasing $\omega(H)$ beyond $\alpha+1$. Otherwise, we add it to $V^{\prime}$.

It is clear that $\omega(H)$ will lie between $\alpha$ and $\alpha+1$. Suppose a vertex $v$ is added to $V^{\prime}$ resulting in $\omega\left(G\left[V^{\prime}\right]\right)>\omega(G)-\alpha$. Let $j=w(v)$. Since $v$ was not added to $V_{H}$, there must be set of vertices of total weight $\alpha+2-j$ adjacent to $v$. Similarly, there must be a set of vertices of total weight $\omega(G)-\alpha+1-j$ adjacent to $v$ in $V^{\prime}$. By our ordering, all of these neighbors of $v$ must form a clique. Thus, we've found a clique of total weight $\omega(G)+3-j>\omega(G)$. This contradicts the definition of $\omega(G)$.

We can now recursively apply our theorem to obtain a decomposition of $G$ into $\left\lceil\frac{\omega(G)}{\alpha}\right]$ interval graphs of clique index bounded by $\alpha+1$. Thus, if we can find aligned $c$-colorings for all $H$ with $\omega(H)=\alpha+1$ then we can color each graph in the decomposition using a disjoint set of colors and obtain an approximate solution.

Corollary 1. Suppose for some $\alpha$, we have a polynomial-time algorithm which can color any 1,2-vertex-weighted interval graph $H$ with $\omega(H) \leq \alpha+1$ using at most $c$ colors. Then we have a polynomial-time $\frac{c}{\alpha}$-approximation algorithm for any 1,2-vertex-weighted interval graph.

We remark that there is a small additive term in our approximation, induced by the ceiling in our division. This can be eliminated by providing a solution with approximation factor $\frac{c}{\alpha}$ whenever $\omega(H) \leq \alpha$. 
We now show how to reduce all interval graphs to a restricted form. This allows us to enforce a problem structure that can be later exploited. We will assume that the corresponding intervals have integer start and end points and are closed towards $-\infty$ and open towards $\infty$. Moreover, we assume that all intervals have non-negative endpoints and we let $B$ denote the latest endpoint of any interval. Thus, the entire instance is bounded within $[0, B]$. We say interval $u$ and interval $v$ are tightly compatible if the startpoint of one is the endpoint of another. We will enforce the following restrictions:

1. Uniform: For $t \in[0, B]$ the total weight of intervals containing $t$ is $\omega(G)$.

2. United: No two tightly compatible intervals have the same weight.

3. Unique: No two weight-1 intervals have identical endpoints.

4. Nested: If two weight-2 intervals intersect, one is contained in the other.

5. Staged: No two weight-2 intervals share a common endpoint.

We will show that given any interval graph $G$, we can produce a new interval graph $G^{\prime}$ of equal clique index and satisfying the above properties such that a coloring of $G^{\prime}$ can be used to efficiently compute a coloring of $G$ (with equal number of colors). We will then describe a polynomial-time algorithm to compute a coloring of $G^{\prime}$ using at most $\frac{4}{3} \omega\left(G^{\prime}\right)$ colors.

Theorem 4. Every 1,2-vertex-weighted interval graph $G$ can be transformed into a 1,2-vertex-weighted interval graph $G^{\prime}$ (in polynomial time) with $\omega\left(G^{\prime}\right)=$ $\omega(G)$ such that all five of the above properties hold. Any aligned c-coloring of $G^{\prime}$ can be used to construct an aligned c-coloring of $G$ (in polynomial time).

Proof. Any interval graph $G$ can be made uniform by adding an appropriate amount of weight-1 intervals during the deficient times. Since this is an extension of $G$, any aligned $c$-coloring directly gives us an aligned $c$-coloring of $G$.

We can make this graph united and unique by merging any tightly compatible weight- 1 intervals, then merging any identical weight- 1 intervals into a weight- 2 interval, then merging any tightly compatible weight- 2 intervals. While we may have $a \chi\left(G^{\prime}\right) \geq a \chi(G)$, an aligned $c$-coloring of $G$ can be recovered from an aligned $c$-covering of $G^{\prime}$ by recording which intervals were merged and assigning colors appropriately.

A united interval graph can be made nested by taking any non-nested, intersecting weight- 2 intervals $u, v$ and replacing them with weight-2 intervals $u \cap v$ and $u \cup v$. We repeat until $G^{\prime}$ is nested. Again, an aligned $c$-coloring of $G$ can be recovered from an aligned $c$-coloring of $G^{\prime}$ if we remember how intervals were spliced; we take the color pairs of $u \cap v$ and of $u \cup v$ and exchange their roles after the splice point. Figure 2 illustrates this process.

We can make an interval graph staged by arbitrarily perturbing one of the intervals and two tightly-compatible weight-1 intervals (selected arbitrarily as well).

We define a stage to be a maximal interval of time during which the number of weight- 2 intervals is constant. An $i$-stage will be a stage during which $i$ weight-2 


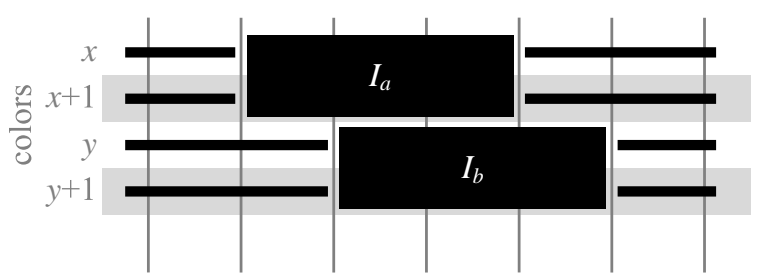

(a)

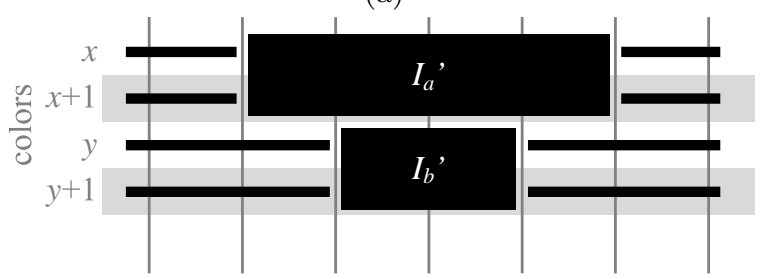

(b)

Fig. 2. Splicing colors. (a) Coloring of a non-nested instance. (b) Coloring of a corresponding nested instance.

intervals are active. Once all the requirements are enforced, there are a number of nice properties we can exploit. First, as time progresses, stages can only increase or decrease by one level. Second, intervals only start and end when the instance is changing stages. Third, when going from an $i$-stage to an $(i+1)$-stage, exactly one weight-2 interval begins and two weight-1 intervals end. Lastly, when going from an $(i+1)$-stage to an $i$-stage, exactly two weight- 1 intervals begin and one weight-2 interval ends.

We will show that $a \chi(10)=12$ for instances satisfying the five properties described (and thus for all instances) and that such colorings can be found in polynomial-time. Our coloring algorithm will color an instance by its stages.

Theorem 5. $a \chi(10)=12$. Moreover, we can construct an aligned 12-coloring in polynomial time.

Proof. We start by removing all weight-2 intervals active during a 1-stage (and thus bordering a 0-stage) and give them color 10 (and 11). The remainder of the instance never has more than total weight 8 of active intervals except for the times where no weight- 2 intervals are active but 10 weight- 1 intervals are active. Call these times (-1)-stages, thus the remainder of our instance now has stages of level -1 through level 4 . Notice that exactly two weight-1 intervals start at the beginning of a (-1)-stage and exactly two end when the (-1)-stage concludes. We will show that we need only 10 additional colors for the remainder of this instance.

Starting from the first stage, greedily assign colors to the active weight-2 intervals, then to the active weight- 1 intervals. This will use no more than 10 colors since the maximum weighted overlap during the first stage is at most 10 . We proceed by coloring from stage to stage. 
When going from an $i$-stage to an $(i-1)$-stage (for $i>0$ ) we simply assign the starting weight- 1 intervals the colors that the terminating weight-2 interval was assigned. When going from a (-1)-stage to a 0 -stage, there are no incoming intervals to color. When going from a 0 -stage to a $(-1)$-stage, we can simply give the two incoming weight-1 intervals the two remaining colors. When going from a 3-stage to a 4-stage at most 3 even colors can be blocked, so the incoming weight-2 interval can choose amongst the remaining 2 even colors. When going from a 2 -stage to a 3 -stage, two even colors are blocked by the continuing weight2 intervals and at most two even colors are blocked by the continuing weight- 1 intervals. This still leaves one open even color for the incoming weight-2 interval.

The interesting cases occur when we go from a 1-stage to a 2-stage and from a 0 -stage to a 1-stage. Consider the former case first. We assume that the weight-2 from the 1 -stage occupies colors $0 / 1$. There are also 4 weight- 1 intervals that conflict with the incoming weight-2. Notice that if we cannot color the weight2 , it must be that each weight- 1 is blocking colors $2 / 3,4 / 5,6 / 7,8 / 9$. Thus, WLOG, we assume that these weight-1s are in colors $2,4,6$, and 8 . Then one of the following cases must hold:

1. All weight-2 intervals seen so far are colored $0 / 1$.

2 . Let $t$ be the ending time of the latest weight- 2 that isn't colored $0 / 1$ (without loss of generality, we'll assume its colored 2/3). At least one of the colors 4 through 9 either have a weight-1 interval starting at $t$ OR are unoccupied between $t$ and $t+1$.

3. None of the colors 4 through 9 have a (weight-1) interval starting at $t$ and all are occupied between $t$ and $t+1$.

In the first case, where no weight-2's have been colored 2 through 9 , it is clear that we can simply swap colors 3 and 4 , then color the incoming weight-2 colors $4 / 5$. Now consider the second case. Let the "culprit" color be the color that either contains a weight- 1 starting at $t$ or is unoccupied at $t$. If the culprit color is either 4,6 or 8 , then we can swap that color with color 3 from time $t$ onwards. This allows us to assign the incoming weight- 2 to the culprit color and its partner. If the culprit color is 5,7 or 9 , then we can swap that color with color 2 from time $t$ onwards. This allows us to color the incoming weight- 2 colors $2 / 3$.

Let us consider the last case. Since just prior to time $t$, we had a weight-2, this means that the stage immediately following time $t$ cannot be a (-1)-stage. In particular, there are 4 colors at time $t$ that either have no assigned active interval or have a weight- 1 starting at $t$. Since case 2 doesn't hold, it follows that these four colors are 0,1,2 and 3. Moreover, since no two weight- 2 intervals share endpoints (by nestedness), color pair $0 / 1$ cannot have an active weight- 2 between times $t-1$ and $t$. Thus, we can swap the contents of colors $0 / 1$ with those of colors $2 / 3$ from time 0 up until $t$. The result is a coloring in which no weight-2 appears in colors 3-10 after time $t-1$. Thus, one of case 1 , case 2 or case 3 with $t$ strictly earlier must hold. If case 3 holds, we can repeat this process only finitely many times (at most $B$ times) until case 1 or case 2 must hold. 
Finally, let us check that we can color when going from a 0-stage to a 1-stage. Note that there must be 6 weight- 1 intervals intersecting the incoming weight- 2 interval. Collectively, these must block all color pairs. Thus, we assume that two of these are in colors $0 / 1$ and the others are colored 2,4,6,8. However, from this point on, we can use the same argument as in the proof of going from a 1-stage to a 2 -stage (this proof did not rely on a weight-2 being in 0/1).

Thus, we can modify our coloring to accomodate all stages in the instance. This proves that $a \chi(10) \leq 12$. Figure 3 gives a tight example showing $a \chi(8) \geq 10$ (which can be extended to show $a \chi(10) \geq 12$ by simply adding intervals).

To color, we do the following for each interval. We retrieve the available colors and their last appearing weight-2 interval and swap some color assignments in linear time. We repeat this process until we can place the interval. However, since there are a linear number of timesteps, we can color a sub-instance in quadratic time. Since we need only record the current color assignment, a linear amount of space suffices.

Theorems 3, 4 and 5 give us a $\frac{4}{3}$-approximation for aligned coloring of general interval graphs. This algorithm decomposes the graph into subgraphs and colors each subgraph independently. Let $n_{i}$ be the size of subgraph $i$. Since we can color each subgraph in time $O\left(n_{i}^{2}\right)$ and space $O\left(n_{i}\right)$, it follows that our algorithm has time complexity $O\left(n^{2}\right)$ and space complexity $O(n)$.

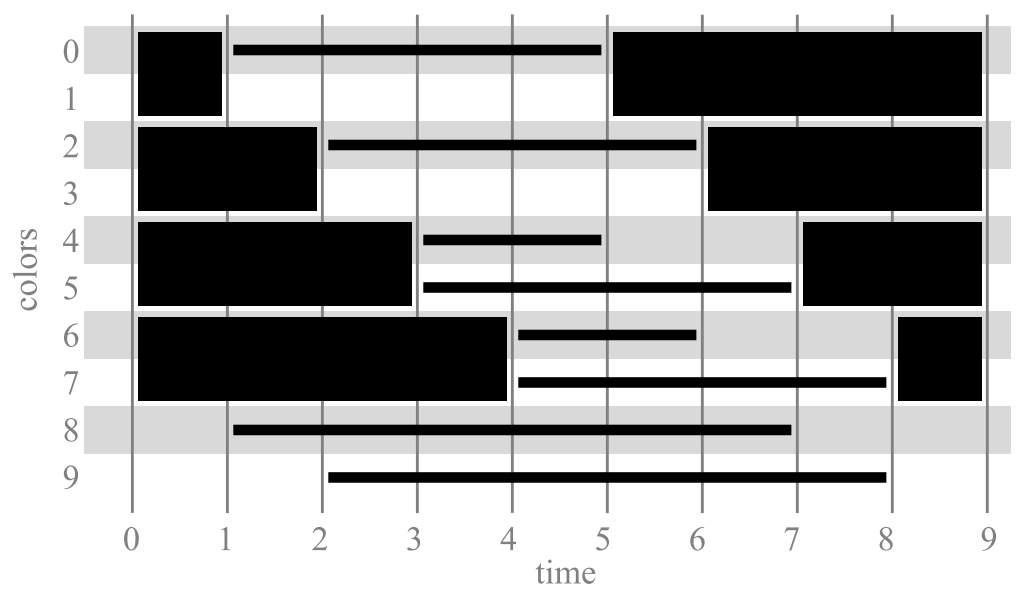

Fig. 3. Example where $a \chi(G)=\omega(G)+2$.

\section{Minimum Spillage}

We now consider the Minimum Spillage Problem defined as follows: 
Definition 2 (Minimum Spillage Problem). We are given a graph $G=$ $(V, E)$ and a number of colors $c$. We wish to select a minimum cardinality set $V^{\prime} \subseteq V$ and a c-coloring $\phi$ of the subgraph $G\left[V-V^{\prime}\right]$ of $G$ induced by $V-V^{\prime}$.

We also consider the Aligned Spillage problem, which asks for a minimum cardinality set $V^{\prime}$ such that $G\left[V-V^{\prime}\right]$ is aligned $c$-colorable. The aligned spillage problem corresponds to the problem of minimizing the number of variables that need to be "spilled" into memory such that the remaining variables can be assigned to the available registers.

\subsection{Unaligned Spillage}

For general graphs, unaligned spillage is NP-Complete since 0 spillage indicates $c$-colorability (thus, solving the unaligned coloring problem). This also indicates that a polynomial-time constant-factor approximation is unlikely to exist. However, given a tree decomposition of width $\tau$, we can use dynamic programming on this decomposition to exhaustively search all possible colorings and get a $O\left(n m(c+1)^{\tau+1}\right)$-time exact algorithm.

Unaligned spillage on chordal graphs is known to be NP-Hard [21]. However, the reduction of [21] is not approximation-preserving and thus does not give a bound on hardness of approximation. We offer an alternative reduction which establishes that unaligned spillage cannot be approximated to within a logarithmic factor.

Theorem 6. The unaligned spillage problem is NP-complete even for chordal graphs. There is no o $(\log |V|)$-approximation unless $N P \subseteq D P T I M E\left(n^{O(\log n)}\right)$.

Proof. This can be shown via a simple approximation-preserving reduction from set cover. Suppose we would like to solve a set cover instance with $n$ elements and $m$ sets. We create a vertex for each of the $m$ sets, and connect all of these vertices into a clique. For each element $x$ we create $m+1-\delta(x)$ vertices, where $\delta(x)$ is the number of sets containing $x$. We connect all of these vertices into a clique. Additionally, we connect each of these vertices to the vertices representing sets containing $x$. It is simple to check that this graph is chordal.

We now ask to color the graph with $c=m$ colors. Suppose that we can compute a partial coloring in polynomial time, which approximately minimizes the number of spilled vertices. We observe that for any vertex representing an element, that vertex and its neighbors form a clique of size exactly $m+1$, from which it follows that for every element, either one of the vertices representing that element is spilled, or one of the vertices representing a set containing that element is spilled. If a vertex representing an element is spilled, we can pick a set containing that element and swap its color for the spilled vertex. This does not increase the number of spilled vertices, and also cannot violate the validity of the coloring. We conclude that we can find a partial coloring with the same number of spilled vertices, where only set vertices are spilled, and for every element one of the sets containing that element has a spilled vertex. Thus the spilled vertices imply a set cover. Conversely, we can spill the vertices of any set cover and get 
a valid coloring (color other vertices greedily). This approximation-preserving reduction from set cover combined with [11] implies the hardness results given.

On a chordal graph, we can obtain a $O(\log |V|)$-approximation by reducing to a set multicover instance. Our elements are supernodes $X_{i}$ of a tree decomposition and our sets are $\left\{X_{i} \mid v \in X_{i}\right\}$ for each $v \in V$. We now wish to find a minimum collection of sets so that each element $X_{i}$ is covered $\left|X_{i}\right|-c$ times. Alternately, we can use dynamic programming to obtain a $O\left(\operatorname{cnm}(\tau+1)^{c}\right)$-time exact algorithm [21].

We can remove the dynamic program runtime's dependence on $\tau$ by doing the following: Fix $\alpha>1$. For each $X_{i}$ in the tree decomposition with $\left|X_{i}\right| \geq \alpha c$, we will spill all of $X_{i}$, then remove every vertex in $X_{i}$ from the tree decomposition. We repeat this process until all supernodes of the tree decomposition have weight less than $\alpha c$. At this point, we can use dynamic programming to find the best coloring in $O\left(n m(\alpha c+1)^{c}\right)$-time.

Theorem 7. The dynamic programming algorithm yields a $\frac{\alpha}{\alpha-1}$-approximation.

Proof. Order the supernodes of the tree decomposition $X_{1}, \ldots, X_{p}$. Let $I=$ $\left\{i_{1}, i_{2}, \ldots, i_{q}\right\}$ be the set of indices corresponding to the $X_{i}$ that were declared spilled. Let $N_{k}=\bigcup_{j<k} X_{i_{j}}$ and $N_{k}^{*}$ be the set of nodes of $N_{k}$ that were spilled by the optimum solution. We would like to show that $\left|N_{q}^{*}\right| \geq \frac{\alpha-1}{\alpha}\left|N_{q}\right|$. We prove this by induction:

Clearly,

$$
\left|N_{1}^{*}\right| \geq\left|X_{1}\right|-c \geq\left|X_{1}\right|\left(1-\frac{1}{\alpha}\right)=\left|N_{1}\right|\left(1-\frac{1}{\alpha}\right)
$$

where the second inequality follows from the fact that $\left|X_{1}\right| \geq \alpha c$.

Now, assume that $\left|N_{j-1}^{*}\right| \geq \frac{\alpha-1}{\alpha}\left|N_{j-1}\right|$. We know that since $X_{j}$ was declared spilled we have $\left|X_{j}-N_{j-1}\right| \geq \alpha c$ which gives $c \leq \frac{\left|X_{j}-N_{j-1}\right|}{\alpha}$. By definition of $N_{j}$, we also have $N_{j}-N_{j-1}=X_{j}-N_{j-1}$. Then notice:

$$
\left|N_{j}^{*}-N_{j-1}^{*}\right| \geq\left|X_{j}-N_{j-1}^{*}\right|-c \geq\left|N_{j}-N_{j-1}\right|\left(1-\frac{1}{\alpha}\right)
$$

Summing this with our inductive hypothesis gives us $\left|N_{j}^{*}\right| \geq\left|N_{j}\right|\left(1-\frac{1}{\alpha}\right)$.

We note that unaligned spillage on interval graphs is polynomial-time solvable by a standard greedy removal of intervals to get a clique index of $c$ [21]. We simply find the smallest time $t$ during which more than $c$ intervals are active, remove the interval with latest endpoint and repeat the process. 


\section{$4.2 \quad$ Aligned Spillage}

The dynamic programming algorithm for chordal graphs is faster than for general graphs because we do not actually need to track the colorings, only the set of spilled nodes. This will not work for aligned spillage, because in an aligned chordal graph the number of colors needed can be greater than the clique index. However, we can still apply the general spillage algorithm in time $O\left(n m(c+1)^{\tau+1}\right)$. We can make use of the technique of theorem 7 to obtain a $\frac{\alpha}{\alpha-1}$-approximation in time $O\left(n m(c+1)^{\alpha c+1}\right)$, an improvement if $\tau \gg c$.

Alternately, we can produce a bicriterion approximation by first reducing the clique number to $c$ by spilling the minimum number of nodes (for an interval graph) or $\log |V|$ times the minimum number of nodes (for a chordal graph), then apply our approximation algorithms for aligned coloring. This yields $\left(\frac{4}{3}, 1\right)$ and $\left(\frac{3}{2}, \log |V|\right)$ approximations for interval and chordal graphs respectively, where an $(\alpha, \beta)$ approximation implies that we color with $\alpha c$ colors while spilling at most $\beta$ times the number of nodes which optimum would have to spill in order to color with $c$ colors.

\section{References}

1. Minwook Ahn, Jooyeon Lee, and Yunheung Paek. Optimistic coalescing for heterogeneous register architectures. SIGPLAN Not., 42(7):93-102, 2007.

2. Preston Briggs. Register allocation via graph coloring. Technical Report TR92-183, Rice University, 1998.

3. Preston Briggs, Keith Cooper, and Linda Torczon. Coloring register pairs. Letters on Programming Languages, 1(1):3-13, 1992.

4. Preston Briggs, Keith D. Cooper, Ken Kennedy, and Linda Torczon. Coloring heuristics for register allocation. SIGPLAN Not., 39(4):283-294, 2004.

5. Preston Briggs, Keith D. Cooper, and Linda Torczon. Improvements to graph coloring register allocation. ACM Trans. Program. Lang. Syst., 16(3):428-455, 1994.

6. David Callahan and Brian Koblenz. Register allocation via hierarchical graph coloring. SIGPLAN Not., 26(6):192-203, 1991.

7. Gregory J. Chaitin. Register allocation and spilling via graph coloring. In $S I G$ PLAN Notices, volume 17, pages 98-105, 1982.

8. Gregory J. Chaitin, Mark A. Auslander, Ashok K. Chandra, John Cocke, Martin E. Hopkins, and Peter W. Markstein. Register allocation via coloring. Computer Languages, 6:47-57, 1981.

9. Frederick Chow and John Hennessy. Register allocation by priority-based coloring. SIGPLAN Not., 19(6):222-232, 1984.

10. Keith Cooper and Linda Torczon. Engineering a Compiler. Morgan Kaufmann, 2003.

11. Uriel Feige. A threshold of $\ln n$ for approximating set cover. Journal of the ACM, 45(4):634-652, 1998.

12. Fanica Gavril. Algorithms for minimum coloring, maximum clique, minimum covering by cliques, and maximum independent set of a chordal graph. Siam Journal of Computing, 1(2):180-187, 1972. 
13. R. Gupta, M. L. Soffa, and T. Steele. Register allocation via clique separators. In PLDI '89: Proceedings of the ACM SIGPLAN 1989 Conference on Programming language design and implementation, pages 264-274, 1989.

14. Sebastian Hack and Gerhard Goos. Optimal register allocation for SSA-form programs in polynomial time. Information Processing Letters, 98(4):150-155, 2006.

15. Subhash Khot. Improved inapproximability results for maxclique, chromatic number and approximate graph coloring. In Proceedings of the 42nd IEEE Symposium on Foundations of Computer Science (FOCS), pages 600-609, 2001.

16. Jonathan K. Lee, Jens Palsberg, and Fernando Magno Quintão Pereira. Aliased register allocation for straight-line programs is NP-complete. In ICALP, pages 680-691, 2007.

17. Fernando Magno Quintão Pereira and Jens Palsberg. Register allocation via coloring of chordal graphs. In Proceedings of the Asian Symposium on Programming Lanugages and Systems (APLAS), pages 315-329, 2005.

18. Fernando Magno Quintão Pereira and Jens Palsberg. Register allocation by puzzle solving. In Proceedings of the 2008 ACM SIGPLAN conference on Programming language design and implementation, pages 216-226, 2008.

19. Donald J. Rose and R. Endre Tarjan. Algorithmic aspects of vertex elimination. In STOC '75: Proceedings of seventh annual ACM symposium on Theory of computing, pages 245-254, 1975.

20. Michael D. Smith, Norman Ramsey, and Glenn Holloway. A generalized algorithm for graph-coloring register allocation. In Proceedings of the ACM SIGPLAN 2004 conference on Programming language design and implementation, pages 277-288, 2004.

21. Mihalis Yannakakis and Fanica Gavril. The maximum $k$-colorable subgraph problem for chordal graphs. Information Processing Letters, 24(2):133-137, 1987. 